\title{
Safety of low- to medium-dose glucocorticoid treatment in rheumatoid arthritis: myths and reality over the years
}

\author{
Tânia Santiago and José António P. da Silva \\ Rheumatology Unit, Centro Hospitalar e Universitário de Coimbra, Coimbra, Portugal
}

Address for correspondence: José António P. da Silva, M.D., Ph.D., Rheumatology Unit, Centro Hospitalar e Univesitário de Coimbra, Praceta Prof. Mota Pinto, 3000-076 Coimbra, Portugal. jdasilva@huc.min-saude.pt

Low- to medium-dose glucocorticoids have been shown to have not only anti-inflammatory but also diseasemodifying properties in rheumatoid arthritis. The evidence for the benefit of its early use in combination with disease-modifying antirheumatic drugs underlines the need for a close evaluation of their risk-benefit ratio. Over time, numerous myths and fears about glucocorticoid toxicity in rheumatoid arthritis have arisen from observational studies, and many concerns have been unduly extrapolated from observations with higher-dose treatment. Furthermore, we cannot exclude the possibility of a powerful effect of bias by indication in these studies. Low- to medium-dose glucocorticoid regimens continued to be evaluated in randomized clinical trials, particularly in early disease, but these studies also have relevant methodological limitations in assessing safety, particularly due to small size and/or short duration. At present, the evidence on which to support clear recommendations about glucocorticoid toxicity remains remarkably weak. A large prospective pragmatic trial dedicated to the toxicity of low-dose glucocorticoids is dearly needed. Meanwhile, adherence to recommendations on standardized methodologies for registration and report of glucocorticoid adverse events is essential for improving our knowledge and competence in the best management of these important medications.

Keywords: glucocorticoids; rheumatoid arthritis; safety; toxicity; observational studies; randomized controlled trials

\section{Introduction}

Glucocorticoids (GCs) are a cornerstone in the treatment of rheumatoid arthritis (RA). In recent years, several high-quality randomized clinical trials have demonstrated their efficacy as diseasemodifying antirheumatic drugs (DMARDs) and a favorable risk-benefit profile in early RA. ${ }^{1,2}$ Despite this, the risk of adverse effects of GCs is constantly underlined, leading to repeated messages that the lowest possible dose should be used for the shortest possible time. Unless these fears of toxicity are well established, we may be unduly depriving patients of valuable benefits from these affordable drugs. The risk and benefit of GCs in RA need, therefore, to be regularly revisited.

Establishing the true risk of adverse effects of GCs is not an easy task: several negative effects attributed to these drugs have been clearly associated with RA itself, especially with severe disease. ${ }^{3}$ This is, for in- stance, the case for osteoporosis, glucose intolerance, and cardiovascular disease. More severe RA is associated with a higher risk of adverse events (AEs) but also with a higher frequency or dose of GC use. GC use is associated with a higher risk of AEs but also with a higher severity of the disease. These complex interplays cannot be totally unraveled in the absence of appropriate randomized trials. Unfortunately, there are only a few randomized clinical trials of GCs in RA, and these are designed and powered for benefit (i.e., they are too short and too small for adequate risk assessment). Observational studies provide most of the evidence available. They are indispensible for identifying relatively rare or late adverse effects but they are entangled in bias by indication that can lead to erroneous conclusions and cannot be completely clarified by statistical manipulation of data.

In this paper, our primary aim was to provide an update of evidence from recent randomized 
controlled trials (RCTs) on the safety of GC treatment in RA. Our secondary aim was to present a critical overview of the numerous problems with both study designs-randomized clinical trials versus nonrandomized observational studies-in assessing the safety of GC treatment in RA.

\section{Evidence from recent clinical trials}

In the last decade, more attention has been given to monitoring and reporting AEs in clinical trials, although scarce evidence was added to the comprehensive review by da Silva et al. published in $2006 .{ }^{4}$ The authors concluded by then that "definitive associations of low-dose GC with many adverse effects remain elusive. The overall fear of GC toxicity in RA, as quoted in textbooks and review articles, is probably overestimated, based on extrapolation from observations with higher dose treatment." We have adopted the definition of low- and medium-dose GCs as $<7.5$ and $<30 \mathrm{mg}$ prednisone equivalent per day, respectively, following a previously proposed nomenclature. ${ }^{5}$ However, some authors would argue that the term low-dose should be restricted to regimes below $5 \mathrm{mg}$ prednisolone equivalent per day, on the basis that such dosages do not induce significant long-term adrenal suppression. ${ }^{6,7}$

Four randomized controlled trials (RCTs) were included in that review: the ARC (Arthritis and Rheumatism Council Low-Dose Glucocorticoid) study, ${ }^{5}$ LDPT (Low-Dose Prednisolone Therapy) study, ${ }^{6}$ Utrecht study, ${ }^{7}$ and WOSERACT (West of Scotland Early Rheumatoid Arthritis Corticosteroid Trial), ${ }^{8}$ with a total of 568 patients observed over 2 years (Table 1). The authors concluded: "Safety data from recent randomized controlled clinical trials of low-dose glucocorticoid treatment in RA suggest that adverse effects associated with these drugs are modest, and often not statistically different from those of placebo."

Since then, three additional clinical trials providing data on the safety of low- to medium-dose GCs over at least 12 months have been published: the BARFOT (Better Anti-Rheumatic FarmacOTherapy) study group, ${ }^{9}$ the CAMERA II (ComputerAssisted Management in Early Rheumatoid Arthritis II) trial, ${ }^{10}$ and a study by Montecucco et al. ${ }^{11}$ (Table 1).

In total, 1274 patients were included in these trials, 624 being exposed to low-dose GCs (5-10 mg prednisone equivalent per day) for 2 years in 514 patients and 1 year in the remaining 110 , totaling 1138 patient-years of GC exposure.

Overall, the toxicity profile of low-dose GCs described in these seven RCTs seems mild and hardly different from that described for placebo, namely regarding new-onset diabetes, osteoporosis, and cardiovascular effects. ${ }^{12}$ New-onset diabetes was reported in six of the seven reviewed trials on low-dose GC AEs. ${ }^{5-10}$ Four $(0.78 \%)$ new cases were identified in the prednisolone group $(n=514)$ during the 2 years of treatment as opposed to three $(0.56 \%)$ cases in the control group $(n=540)$.

Bone mineral density (BMD) loss over 2 years was not significantly different from that with placebo in these trials (Table 2). ${ }^{5,7-9}$ Fracture incidences were small and similar between the groups, when reported. However, in the Utrecht study, GC treatment was associated with a doubling of the incidence of radiological vertebral fractures, although this did not reach statistical significance. ${ }^{7,13}$ It is important to emphasize that studies were too small to evaluate fracture incidence. Only one of the studies does not describe their intervention regarding osteoporosis prevention. ${ }^{5}$ In the LDPT study, ${ }^{6}$ calcium and vitamin D were permitted, as was estrogen replacement therapy. In the Utrecht ${ }^{7,14}$ and BARFOT studies, ${ }^{9}$ all participants received a $500-$ and 1000 -mg calcium daily supplement, respectively.

In the WOSERACT study, ${ }^{8}$ the prescription of bisphosphonates, which was left to physicians' discretion, increased in both groups from $2 \%$ to $13 \%$ (prednisolone group) and from $1 \%$ to $5 \%$ (placebo), at baseline and after 2 years, respectively. In addition, the prescription of any osteoporosis treatment increased 15-30\% (prednisolone group) and 6-12\% (placebo), at baseline and after 2 years, respectively. In the CAMERA-II study, ${ }^{10}$ all patients received bisphosphonate, calcium $500 \mathrm{mg}$, and vitamin D 400 IU daily. In the study by Montecucco et al., ${ }^{11} \mathrm{pa}-$ tients were also given prophylactic treatment with oral calcium and vitamin $\mathrm{D}$.

Similarly, toxicity data from the seven reviewed trials have been very reassuring about the effect on cardiovascular events. For example, low-dose GCs in the prednisolone group had no significant effect on blood pressure in any of the trials. ${ }^{5-8}$ Toxicity data on lipid profile were reported in only one of the seven trials. According to the described methodology in the WOSERACT study, participants had total cholesterol and HDL cholesterol measured at the 
Table 1. Characteristics of the randomized controlled trials using low-dose GCs in RA

\begin{tabular}{|c|c|c|c|c|c|c|c|}
\hline Study & $\begin{array}{l}\text { Year of } \\
\text { first pub- } \\
\text { lication }\end{array}$ & $\begin{array}{c}\text { RA } \\
\text { duration } \\
\text { at entry } \\
\text { (years) }\end{array}$ & $\begin{array}{l}\text { Number } \\
\text { of } \\
\text { patients, } \\
\text { P-group }\end{array}$ & $\begin{array}{l}\text { Prednisolone } \\
\text { dose (mg/day) }\end{array}$ & $\begin{array}{l}\text { Duration } \\
\text { of the } \\
\text { trial } \\
\text { (years) }\end{array}$ & $\begin{array}{l}\text { Associated } \\
\text { DMARDs }\end{array}$ & $\begin{array}{c}\text { Number of withdrawals in } \\
\text { P-group versus } \\
\text { NoP-group, } n(\%)\end{array}$ \\
\hline ARC study ${ }^{5}$ & 1995 & $<2$ & 61 & $7.5^{a}$ & 2 & Various & $1(1.6)$ vs. 5 (7.5) \\
\hline LDPT study ${ }^{6}$ & 2000 & $<2$ & 93 & $5^{a}$ & 2 & $\begin{array}{l}\text { IM gold or } \\
\text { MTX }\end{array}$ & $\begin{array}{l}\text { Safety, } 10(10.6) \text {, drug } \\
\text { failure, } 6(6.4) \text {, others, } \\
29(30.9) \text { vs. safety, } 12 \\
\text { (12.3), drug failure, } \\
\text { 13(13.2), others, } 19 \\
\text { (19.4) }\end{array}$ \\
\hline Utrecht study $^{7}$ & 2002 & $<1$ & 40 & $10^{a}$ & 2 & SSZ rescue & $4(10.0)$ vs. $6(14.6)$ \\
\hline WOSERACT ${ }^{8}$ & 2004 & Median 1 & 84 & $7^{a}$ & 2 & SSZ & $6(7.1)$ vs. $2(2.4)$ \\
\hline BARFOT study ${ }^{9}$ & 2005 & $<2$ & 119 & $7.5^{a}$ & 2 & MTX or SSZ & $\begin{array}{l}26 \text { (21.8) (prednisolone } \\
\text { was judged to be the } \\
\text { cause in } 5 \text { patients) vs. } \\
24(18.3)\end{array}$ \\
\hline $\begin{array}{l}\text { CAMERA-II } \\
\text { study }^{10}\end{array}$ & 2012 & $<2$ & 117 & $10^{a}$ & 2 & MTX & 16 (14) vs. 20 (17) \\
\hline $\begin{array}{l}\text { Montecucco } \\
\text { et al. }^{11}\end{array}$ & 2012 & $<2$ & 110 & $6.25^{b}$ & 1 & MTX & $\begin{array}{l}\text { No data “... In general, } \\
\text { patients on GC were } \\
\text { more likely to withdraw } \\
\text { the treatment protocol." }\end{array}$ \\
\hline
\end{tabular}

Note: DMARDs, disease-modifying antirheumatic drugs; IM, intramuscular; MTX, methotrexate; SSZ, sulphasalazine; P-group, prednisolone group; NoP-group, nonprednisolone group.

${ }^{a}$ Stable dose throughout the study.

${ }^{b} 12.5 \mathrm{mg} /$ day for 2 weeks tapered to $6.25 \mathrm{mg} /$ day.

start and end of 2 years. ${ }^{8}$ Prednisone had no significant effect on the lipid levels of those patients in whom it was measured. Furthermore, carotid atherosclerosis and endothelial function were assessed by ultrasonography in a subset of 67 patients from the BARFOT study, 5 years after the start of their first DMARD: 21 patients had been treated with $7.5 \mathrm{mg}$ prednisolone daily for 2 years and 13 patients continuously for 5 years before vascular evaluation, in comparison with 33 nonexposed patients. ${ }^{15}$ The authors found that low-dose prednisolone did not influence endothelial function and atherosclerosis in RA.

On the other hand, the toxicity profile described in these seven RCTs suggests that low-dose GCs are associated with an increase of body weight gain and glaucoma over 2 years. The increase in body weight was significantly higher in the prednisolone group than in the placebo group in three studies, ${ }^{6,7,10}$ an effect that was not found in two other studies. ${ }^{5,8}$ In a subanalysis of the CAMERA-II study, Jurgens et al. concluded that part of the difference in weight gain between the groups was due to an earlier and better control of disease activity with prednisone. ${ }^{16}$ In fact, weight gain under GC treatment may, in part, be because of the recovery of weight lost due to the catabolic state associated with high disease activity. ${ }^{16}$ Conversely, decreasing disease activity might be an expected result in increased physical mobility, which could promote weight loss. GCs are endowed with direct effects on glucose and fat metabolism. Further studies are needed to fully clarify the mechanisms underlying body weight change in RA under GC treatment.

Ocular AEs were reported in only three of the seven trials. According to the description of 
Table 2. Effect of glucocorticoid treatment on lumbar and hip bone mineral density in RA in the prednisolone group versus placebo group

\begin{tabular}{|c|c|c|c|c|c|c|}
\hline & \multicolumn{2}{|c|}{$\begin{array}{c}\text { Number of participants } \\
\text { included in the BMD } \\
\text { analysis }\end{array}$} & \multirow{2}{*}{$\begin{array}{l}\text { Follow-up } \\
\text { - period } \\
\text { (years) }\end{array}$} & \multirow{2}{*}{ BMD Unit } & \multirow[b]{2}{*}{ Lumbar site } & \multirow[b]{2}{*}{ Hip site } \\
\hline & P-group & NoP-group & & & & \\
\hline ARC study ${ }^{5}$ & 11 & 10 & 2 & $\begin{array}{l}\% \text { BMD } \\
\text { loss }\end{array}$ & $\begin{array}{l}\text { After one and two } \\
\text { years: } \\
1.61 \pm 4.98 \text { and } 2.96 \\
\pm 5.59 \text { vs. } 2.27 \pm \\
5.54 \text { and } 1.29 \pm \\
4.64^{a} \\
\text { Not statistically } \\
\text { different }\left({ }^{\star} 1\right)\end{array}$ & $\begin{array}{l}\text { After one and two } \\
\text { years: } \\
2.16 \pm 7.07 \text { and } \\
1.19 \pm 3.16 \text { vs. } \\
0.56 \pm 5.62 \text { and } \\
4.02 \pm 2.45^{a}(P= \\
0.04 \text { at two years }) \\
\left({ }^{\star} 1\right) .\end{array}$ \\
\hline LDPT study $^{6}$ & 23 & 23 & 2 & NA & $\begin{array}{l}\text { “. . slightly greater dec1 } \\
\text { prednisolone group" } \\
\text { interpretation of the }\end{array}$ & $\begin{array}{l}\text { rease in BMD in the } \\
\text {... "a reliable } \\
\text { data is not possible." }\end{array}$ \\
\hline Utrecht study ${ }^{7,14}$ & 32 & 32 & 3 & T-score & $\begin{array}{l}\text { At baseline, } 2 \text { years, } \\
\text { and } 3 \text { years: } \\
-0.8 \pm 0.3 \text { to }-1.1 \\
\pm 0.3 \text { to }-1.1 \pm 0.3 \\
\text { vs. }-0.7 \pm 0.3 \text { to } \\
-0.6 \pm 0.3 \text { to } \\
-0.6 \pm 0.3^{b} \\
\text { Not statistically } \\
\text { significant intra- or } \\
\text { intergroups }\left({ }^{*} 1\right)\end{array}$ & $\begin{array}{l}\text { At baseline, } 2 \text { years, } \\
\text { and } 3 \text { years: } \\
-1.8 \pm 0.2 \text { to }-1.9 \\
\pm 0.2 \text { to }-1.8 \pm 0.2 \\
\text { vs. }-1.9 \pm 0.2 \text { to } \\
-1.9 \pm 0.2 \text { to }-1.9 \\
\pm 0.2^{b} \\
\text { Not statistically } \\
\text { significant intra- or } \\
\text { intergroups }\left({ }^{*} 1\right)\end{array}$ \\
\hline WORSERACT $^{8}$ & 84 & 83 & 2 & $\mathrm{~g} / \mathrm{cm}^{2}$ & $\begin{array}{l}\text { At baseline and } \\
\text { after two years: } \\
1.079 \text { and } 1.073 \mathrm{vs.} \\
1.157 \text { and } 1.280^{b} \\
\text { Not statistically } \\
\text { significant }\end{array}$ & $\begin{array}{l}\text { At baseline and } \\
\text { after two years: } \\
0.899 \text { and } 0.881 \text { vs. } \\
0.927 \text { and } 0.911^{b} \\
\text { Not significantly } \\
\text { different }\end{array}$ \\
\hline BARFOT study $^{9}$ & & & 2 & $\mathrm{~g} / \mathrm{cm}^{2}$ & $\begin{array}{l}\text { At baseline and after } \\
2 \text { years: } \\
1.14 \pm 0.16 \text { and } 1.11 \\
\pm 0.16 \text { vs. } 1.17 \pm \\
0.21 \text { and } 1.16 \pm \\
0.21^{b} \\
\text { Not statistically } \\
\text { significant }\end{array}$ & $\begin{array}{l}\text { At baseline and } \\
\text { after } 2 \text { years: } \\
0.91 \pm 0.15 \text { and } \\
0.90 \pm 0.16 \text { vs. } 0.90 \\
\pm 0.15 \text { and } 0.87 \pm \\
0.15^{b} \\
\text { Not statistically } \\
\text { significant }\end{array}$ \\
\hline CAMERA-II study ${ }^{10,13}$ & 85 & 94 & 2 & $\mathrm{~g} / \mathrm{cm}^{2}$ & $\begin{array}{l}\text { At baseline, and } \\
\text { after } 1 \text { and } 2 \text { years: } \\
1.13,1.16 \text { and } 1.15 \\
\text { (not statistically } \\
\text { significant) vs. } 1.11 \\
\text { and } 1.14 \text { and } 1.15^{b} \\
(P=0.01)\end{array}$ & $\begin{array}{l}\text { "The sBMD in the left } \\
\text { hip did not change } \\
\text { significantly during } \\
\text { the } 2 \text { years of } \\
\text { treatment." }\end{array}$ \\
\hline
\end{tabular}




\begin{tabular}{|c|c|c|c|c|c|c|}
\hline & \multicolumn{2}{|c|}{$\begin{array}{c}\text { Number of participants } \\
\text { included in the BMD } \\
\text { analysis }\end{array}$} & \multirow{2}{*}{$\begin{array}{l}\text { Follow-up } \\
\text { period } \\
\text { (years) }\end{array}$} & \multirow[b]{2}{*}{ BMD Unit } & \multirow[b]{2}{*}{ Lumbar site } & \multirow[b]{2}{*}{ Hip site } \\
\hline & P-group & NoP-group & & & & \\
\hline Montecucco et al. ${ }^{11}$ & NA & NA & NA & NA & NA & NA \\
\hline \multicolumn{7}{|c|}{$\begin{array}{l}\text { Note: }{ }^{\star} 1 \text { derived from da Silva et al. }{ }^{4} \text { (web-only additional). } \\
\text { NoP-group, nonprednisolone group; NA, not applicable. } \\
{ }^{a} \text { Mean } \pm \text { SD percentage reductions. } \\
{ }^{b} \text { Mean } \pm \text { SD. }\end{array}$} \\
\hline
\end{tabular}

methodology, the LDPT seems to have adopted the most stringent ocular evaluation protocol: all participants had an eye examination at the start and end of 2 years. ${ }^{6}$ Three news cases of glaucoma were identified in the prednisone group $(n=93)$ during the 2 years of treatment as opposed to none in the control group $(n=96)$. The incidence of cataracts was of five and six cases, respectively.

Overall, therefore, the conclusions concerning safety of low-dose GC treatment in RA in 2013 are approximately the same as those of 2006: RCTs indicate that the toxicity of low-to-medium doses of GCs in RA used for 2 years is mild and not statistically different from placebo.

\section{Where is the evidence (for fear) coming from?}

Most of the quoted evidence comes from observational studies. The contrast between the two sources of evidence (observational studies and RCTs) is highlighted by the recent systematic review by Dixon et al. ${ }^{17}$ They compiled data from 21 RCTs (including 1026 GC-treated patients with RA) and 42 observational studies, from which we will use infections as the leading example. The estimated relative risk (RR) of infection associated with GC therapy differed significantly between the RCTs (RR 0.97; 95\% CI, 0.69-1.36) and the observational studies (RR 1.67; 95\% CI, 1.49-1.87). There was considerable heterogeneity in the observational studies, which was attributed to differing GC doses, cumulative exposure, time-varying exposure, cotherapy, comorbidity, recruitment methods, outcome, and bias (particularly publication bias). ${ }^{17,18}$ Having performed such a thorough review of the evidence, one must conclude that the true risk of infection associated with GC treatment in RA remains uncertain. RCTs are not only of limited dimension and duration but they have also been designed for efficacy and frequently demonstrate an inconsistent reporting of safety outcomes. Conversely, observational studies are markedly heterogeneous and cannot be stripped of the inherent bias by indication.

Studies exploring the association between GCs and osteoporosis also reveal conflicting findings. A meta-analysis from 2004 included approximately 42,000 men and women drawn from seven prospective population observational studies. ${ }^{19}$ The RR of hip fracture for prior use of GCs, without adjustment for BMD, was 2.31 (95\% CI, 1.7-3.2) and with adjustment for BMD, the RR was 2.25 (95\% CI, 1.6-3.2). Interestingly, RA was also associated with an independent increased risk of osteoporotic hip fracture (RR 1.56; 95\% CI, 1.2-2.0), which persisted after adjustment for GC use (adjusted RR 1.46; 95\% CI, 1.1-1.9). ${ }^{20} \mathrm{~A}$ second study included 30,262 RA patients, aged $>40$ years, from the British General Practice Research Database, of whom 2460 experienced a fracture during the mean 7.6 years of follow-up. ${ }^{21}$ The authors found that the risk of fracture in patients with RA remained significantly elevated after excluding patients who had taken oral GCs at any time during the period of follow-up (adjusted RR for clinical osteoporotic fracture 1.3; 95\% CI, 1.2-1.4) (adjusted RR for hip fracture 1.7; 95\% CI, 1.5-2.0). These data highlight that RA is, itself, a risk factor for osteoporotic fracture, irrespective of GC use, thus confounding the argument. BMD was systematically assessed in four of the seven randomized trials described in Table 1. None of these studies 
found statistically significant effects of prednisone on BMD when compared with placebo. Fracture incidences were small and similar between the groups, when reported. ${ }^{13}$

Similarly, studies exploring the association between GCs and hyperglycemia/diabetes reveal conflicting findings. A recent report indicates that GC users have a roughly $30 \%$ increased risk of diabetes in comparison to nonusers. ${ }^{22}$ However, in a detailed study of glucoregulation, Hoes et al. ${ }^{23}$ reached considerably different conclusions. The authors measured glucose tolerance, insulin sensitivity, and $\beta$ cell function in two RA populations (58 chronic GC users and 82 GC-naive patients) and in healthy controls, with no known type 2 diabetes mellitus. Chronic GC users and GC-naive RA patients presented similar metabolic parameters, with decreased insulin sensitivity and $\beta$ cell function in comparison to controls. Cumulative doses of GCs had a negative effect on glucose tolerance state and insulin sensitivity. The results highlight a complex interplay of three factors: (1) the RA-induced proinflammatory state negatively affects glucose metabolism; (2) GCs downregulate disease activity, which may diminish this effect, but (3) GC itself impairs glucose metabolism. ${ }^{24}$ Similar interactions probably occur within other metabolic pathways, including osteoporosis and cardiovascular events.

As mentioned above, data from randomized trials are quite reassuring in this respect; no relevant differences in the number of cases of new-onset diabetes were seen in either of the trials. The Utrecht study found the least favorable results; a significant increase in mean (SD) fasting glucose was seen in the prednisone group (from $5.1(0.6)$ at baseline to $5.9(1.9) \mathrm{mmol} / \mathrm{L}$ at 2 years, $P<0.01){ }^{7}$ On the other hand, in the CAMERA-II study performed by the same group, $10 \mathrm{mg}$ prednisone daily did not lead to higher glucose levels or an increased incidence of diabetes over 2 years of treatment. ${ }^{10}$

Conflicting results and a lack of clear data are also the rule for other potential AEs, including, for example, peptic ulcer, hypertension, dyslipidemia, cardiovascular disease, and adrenal insufficiency. In fact, as in 2006, we may conclude that few of the commonly held beliefs about the incidence, prevalence, and effects (of low-dose GC AEs) are supported by clear scientific evidence.

It may seem that we are unduly downplaying the quality of evidence provided by observational stud- ies. A closer look at one such example is warranted. On the basis of a retrospective database analysis of RA patients, ${ }^{25}$ the authors concluded that current or past use of GCs was associated with a higher rate of a variety of poor outcomes, including mortality, work disability, and total joint replacement. It was concluded that the longer the GC treatment (up to more than 40 years of use in this study), that is, the higher the cumulative dose, the higher the rate of poor outcomes. Such conclusions lead the reader to believe that GCs are the cause of these poor outcomes. Furthermore, virtually all negative risk factors found from the data had a higher prevalence among GC users than in nonusers. This includes smoking, educational level, male gender, but also, for example, disability, comorbidity, and number of DMARDs used. Obviously, the possibility that GC use is a marker of more severe disease may itself contribute to poor outcomes. However, this is scarcely underlined in the abstract conclusion: "Corticosteroid use is also associated with adverse long-term outcomes, but the ability to discern causal associations is severely limited by confounding by indication." Similar comments could be made about virtually all observational studies. Of course, efforts are done in most such studies to statistically adjust for confounders but it is virtually impossible to guarantee that a retrospective analysis of nonrandomized observations is free from bias. ${ }^{26}$

The paradoxical finding of many of these studies is the confirmation that many patients with RA use GCs, despite the fact that their side effects are so commonly highlighted in the literature. Certainly, rheumatologists have different practices in prescribing GCs to their patients, and these differences may be due to a variety of reasons, including, for example, economic, educational, demographic characteristics of the patient populations, and patients' attitudes. However, the decision of whether to use GCs will have a strong influence on the course of RA, the risk of toxicity, and the cost of treatment. The foundations of such decisions need, therefore, to be scrutinized.

\section{Observational studies versus randomized clinical trials}

RCTs are the gold-standard study design for efficacy. None of the available RCTs of GCs in RA were designed to assess toxicity of GCs and the attention dedicated to toxicity seems to be, with few 
exceptions, very limited. Furthermore, their size and duration are too small to allow for definite conclusions regarding safety. As a result of their small size and small number of events, the absence of statistical significance for the observed numerical differences in the incidence of some AEs cannot be considered proof of the absence of an effect. In the carefully conducted CAMERA II study, only seven serious AEs occurred over 2 years in 236 patients, two of them under prednisone treatment and five under placebo. ${ }^{10}$ These results are reassuring but need to be interpreted with caution, both because of the number of patients and the duration of follow-up. ${ }^{10}$

We also need to recognize that the tight inclusion and exclusion criteria of RCTs limit the generalizability of the results. Their applicability can also be questioned by the fact that a fixed dose is given to patients irrespective of their clinical response, as opposed to the flexible regimen used in clinical practice. Some authors also argue that the available clinical trials use higher GC doses than are really necessary to achieve the desired clinical results, thus contributing to unnecessary toxicity. ${ }^{27}$ In a recent retrospective study, minimal long-term AEs were observed in patients who have taken a very low dose of GCs for more than 10 years. ${ }^{28}$ Certainly, the limitations of the available clinical trials need to be taken into account when analyzing the evidence: absence of evidence for serious toxicity is not the same as evidence that such toxicity is absent.

Observational studies do provide the opportunity to understand the likelihood of GC AEs with real-life dose patterns in patients with different characteristics and allow for a much longer observation in larger samples of the population. However, the limitations of these nonrandomized observational studies cannot be ignored either. The potential importance of this source of bias can be illustrated with published data from the Multinational Etoricoxib and Diclofenac Arthritis Long-Term (MEDAL) program, where cardiovascular safety of etoricoxib was compared to that of diclofenac. ${ }^{29}$ Patients were randomized to one of the treatment arms and stratified according to cardiovascular risk factors. The appropriate reading of the data shows that there are no significant differences between the treatment arms either in the presence or absence of low-dose aspirin. However, the rate of cardiovascular events is higher in patients taking low-dose aspirin than in those not taking this medication, irrespective of being ex- posed to etoricoxib or diclofenac during the trial. The reason for this is simple: patients with high cardiovascular risk were selected for aspirin treatment. This resulted in a reduction of the rate of events but not to the level of the low-risk group. However, if the MEDAL population was approached from an observational perspective, the conclusion might be that aspirin is associated with an increased risk of cardiovascular events.

Robust observational studies would require detailed information on GC exposure through time (start and stop dates, changing dose, oral and parenteral therapy, and adherence), outcomes, and a thorough accounting of all confounders including disease severity. Moreover, patients with severe disease could not be left untreated if they needed GCs and bias would be inevitable.

In summary, both RCTs and observational studies have significant limitations in their ability to establish the safety of GCs. The former tends to underestimate the true risk, whereas the opposite occurs with the latter. Definite conclusions will not be possible unless a properly sized, pragmatic randomized controlled trial is performed.

\section{Conclusion}

Given the numerous problems with both study designs in assessing safety with GC treatment, how can the evidence be best summarized? On the current status of evidence, all we can state is that there is no evidence that low-dose GCs are associated with significant toxicity in early RA over 2 years, besides weight gain and probably glaucoma. This is not to say that we have evidence that there is no significant toxicity.

Definite conclusions about the safety of GCs require RCTs with sufficient dimension and duration, and with appropriate standardization in the definition and monitoring of AEs. ${ }^{3}$ Such a trial may never happen at the hands of the pharmaceutical industry but its need cannot be ignored by the clinical and scientific community. How can we continue to so frequently use medications that we know so little about? Incredible as it may seem, the toxicity of low-dose GCs remains an orphan condition in rheumatology.

Meanwhile, it would be of great assistance if new trials adhered to standardized procedures for the monitoring and reporting of GC-related AEs, such as those recommended by the European League 
Against Rheumatism GC task force. ${ }^{30}$ Research to improve the existing tools to measure some of these side effects (such as cutaneous and psychological side effects) is greatly needed.

\section{Conflicts of interest}

\section{The authors declare no conflicts of interest.}

\section{References}

1. Jacobs, J.W. et al. 2006. Followup radiographic data on patients with rheumatoid arthritis who participated in a twoyear trial of prednisone therapy or placebo. Arthritis Rheum. 54: 1422-1428.

2. Kirwan, J.R. et al. 2007. Effects of glucocorticoids on radiological progression in rheumatoid arthritis. Cochrane Database Syst. Rev. CD006356.

3. van der Goes, M.C. et al. 2010. Monitoring adverse events of low-dose glucocorticoid therapy: EULAR recommendations for clinical trials and daily practice. Ann. Rheum. Dis. 69: 1913-1919.

4. Da Silva, J.A. et al. 2006. Safety of low dose glucocorticoid treatment in rheumatoid arthritis: published evidence and prospective trial data. Ann. Rheum. Dis. 65: 285-293.

5. Kirwan, J.R. and The Arthritis and Rheumatism Council Low-Dose Glucocorticoid Study Group. 1995. The effect of glucocorticoids on joint destruction in rheumatoid arthritis. N. Engl. J. Med. 333: 142-146.

6. Wassenberg, S. et al. 2005. Very low-dose prednisolone in early rheumatoid arthritis retards radiographic progression over two years: a multicenter, doubleblind, placebo-controlled trial. Arthritis Rheum. 52: 3371-3380.

7. van Everdingen, A.A. et al. 2002. Low-dose prednisone therapy for patients with early active rheumatoid arthritis: clinical efficacy, disease-modifying properties, and side effects: a randomized, double-blind, placebo-controlled clinical trial. Ann. Intern. Med. 136: 1-12.

8. Capell, H.A. et al. 2004. Lack of radiological and clinical benefit over two years of low dose prednisolone for rheumatoid arthritis: results of a randomised controlled trial. Ann. Rheum. Dis. 63: 797-803.

9. Svensson, B. et al. 2005. Low-dose prednisolone in addition to the initial disease-modifying antirheumatic drug in patients with early active rheumatoid arthritis reduces joint destruction and increases the remission rate: a two-year randomized trial. Arthritis Rheum. 52: 3360-3370.

10. Bakker, M.F. et al. 2012. Low-dose prednisone inclusion in a methotrexate-based, tight control strategy for early rheumatoid arthritis: a randomized trial. Ann. Intern. Med. 156: 329-339.

11. Montecucco, C. et al. 2012. Low-dose oral prednisone improves clinical and ultrasonographic remission rates in early rheumatoid arthritis: results of a 12-month open-label randomised study. Arthritis Res. Ther. 14: R112.

12. Santiago, T. \& J.A. da Silva. 2014. Safety of glucocorticoids in rheumatoid arthritis: evidences from recent clinical trials. Neuroimmunomodulation. In Press.
13. van der Goes, M.C. et al. 2013. Are changes in bone mineral density different between groups of early rheumatoid arthritis patients treated according to a tight control strategy with or without prednisone if osteoporosis prophylaxis is applied? Osteoporos. Int. 24: 1429-1436.

14. van Everdingen, A.A. et al. 2003. Low-dose glucocorticoids in early rheumatoid arthritis: discordant effects on bone mineral density and fractures? Clin. Exp. Rheumatol. 21: 155-160.

15. Hafstrom, I. et al. 2007. Effects of low-dose prednisolone on endothelial function, atherosclerosis, and traditional risk factors for atherosclerosis in patients with rheumatoid arthritis-a randomized study. J. Rheumatol. 34: 18101816.

16. Jurgens, M.S. et al. 2013. Increase of body mass index in a tight controlled methotrexate-based strategy with prednisone in early rheumatoid arthritis: side effect of the prednisone or better control of disease activity? Arthritis Care Res. (Hoboken). 65: 88-93.

17. Dixon, W.G., S. Suissa \& M. Hudson. 2011. The association between systemic glucocorticoid therapy and the risk of infection in patients with rheumatoid arthritis: systematic review and meta-analyses. Arthritis Res. Ther. 13: R139.

18. Widdifield, J. et al. 2013. Serious infections in a populationbased cohort of 86,039 seniors with rheumatoid arthritis. Arthritis Care Res. (Hoboken) 65: 353-361.

19. Kanis, J.A. et al. 2004. A meta-analysis of prior corticosteroid use and fracture risk. J. Bone Miner. Res. 19: 893-899.

20. Kanis, J.A. et al. 2005. Assessment of fracture risk. Osteoporos. Int. 16: 581-589.

21. van Staa, T.P. et al. 2006. Clinical assessment of the longterm risk of fracture in patients with rheumatoid arthritis. Arthritis Rheum. 54: 3104-3112.

22. Solomon, D.H. et al. 2010. Risk of diabetes among patients with rheumatoid arthritis, psoriatic arthritis and psoriasis. Ann. Rheum. Dis. 69: 2114-2117.

23. Hoes, J.N. et al. 2011. Glucose tolerance, insulin sensitivity and beta-cell function in patients with rheumatoid arthritis treated with or without low-to-medium dose glucocorticoids. Ann. Rheum. Dis. 70: 1887-1894.

24. Buttgereit, F. 2011. Do the treatment with glucocorticoids and/or the disease itself drive the impairment in glucose metabolism in patients with rheumatoid arthritis? Ann. Rheum. Dis. 70: 1881-1883.

25. Caplan, L. et al. 2007. Corticosteroid use in rheumatoid arthritis: prevalence, predictors, correlates, and outcomes. J. Rheumatol. 34: 696-705.

26. Boers, M. 2007. Studying the benefit/risk ratio of glucocorticoids in rheumatoid arthritis. J. Rheumatol. 34: 661663.

27. Pincus, T. et al. 2009. Efficacy of prednisone $1-4 \mathrm{mg} /$ day in patients with rheumatoid arthritis: a randomised, doubleblind, placebo controlled withdrawal clinical trial. Ann. Rheum. Dis. 68: 1715-1720.

28. Pincus, T. et al. 2013. Decline of mean initial prednisone dosage from 10.3 to $3.6 \mathrm{mg} /$ day to treat rheumatoid arthritis between 1980 and 2004 in one clinical setting, with longterm effectiveness of dosages less than $5 \mathrm{mg} /$ day. Arthritis Care Res. (Hoboken) 65: 729-736. 
29. Cannon, C.P. et al. 2006. Cardiovascular outcomes with etoricoxib and diclofenac in patients with osteoarthritis and rheumatoid arthritis in the Multinational Etoricoxib and Diclofenac Arthritis Long-term (MEDAL) programme: a randomised comparison. Lancet 368: 17711781.
30. van der Goes, M.C. et al. 2010. Patient and rheumatologist perspectives on glucocorticoids: an exercise to improve the implementation of the European League Against Rheumatism (EULAR) recommendations on the management of systemic glucocorticoid therapy in rheumatic diseases. Ann. Rheum. Dis. 69: 1015-1021. 